\title{
Evolutionary study of potentially zoonotic hepatitis E virus genotype 3 from swine in Northeast Brazil
}

\author{
Edmilson Ferreira de Oliveira-Filho, ${ }^{1,2}+$, Debora RL dos Santos ${ }^{3}$, Ricardo Durães-Carvalho', \\ Adalúcia da Silva ${ }^{1}$, Gustavo Barbosa de Lima ${ }^{4}$, Antônio Fernando B Batista Filho ${ }^{5}$, \\ Lindomar J Pena', Laura HVG Gil ${ }^{1}$
}

${ }^{1}$ Fundação Oswaldo Cruz-Fiocruz, Instituto Aggeu Magalhães, Departamento de Virologia, Recife, PE, Brasil

${ }^{2}$ Charité-Universitätsmedizin Berlin, Corporate Member of Freie Universität Berlin, Humboldt-Universität zu Berlin, and Berlin Institute of Health, Institute of Virology, Berlin, Germany

${ }^{3}$ Universidade Federal Rural do Rio de Janeiro, Instituto de Veterinária, Seropédica, RJ, Brasil

${ }^{4}$ Fundação Oswaldo Cruz-Fiocruz, Instituto Aggeu Magalhães, Departamento de Microbiologia, Recife, PE, Brasil

${ }^{5}$ Universidade Federal Rural de Pernambuco, Departamento de Medicina Veterinária, Recife, PE, Brasil

Hepatitis E virus (HEV), an emerging virus associated with acute hepatic disease, leads to thousands of deaths worldwide. HEV has already been reported in Brazil; however, there is a lack of epidemiological and molecular information on the genetic variability, taxonomy, and evolution of HEV. It is thus unclear whether hepatitis E is a neglected disease in Brazil or it has low relevance for public health in this country. Here, for the first time, we report the presence of HEV in Northeast Brazil. A total of 119 swine faecal samples were screened for the presence of HEV RNA using real-time polymerase chain reaction (RT-PCR) and further confirmed by conventional RT-PCR; among these, two samples were identified as positive. Molecular evolution analyses based on capsid sequences revealed that the samples had close proximities to HEV sequences belonging to genotype 3 and were genetically related to subtype 3 f isolated in humans. Parsimony ancestral states analysis indicated gene flow events from HEV cross-species infection, suggesting an important role of pig hosts in viral spillover. HEV's ability for zoonotic transmission by inter-species host switching as well as its possible adaptation to new animal species remain important issues for human health.

Key words: zoonotic hepatitis E virus - swine - genotype 3

Hepatitis E is an emerging zoonotic disease caused by the hepatitis E virus (HEV). HEV is a member of the genus Orthohepevirus A and can be divided into eight major genotypes ${ }^{(1)}$ and 43 subtypes (assigned and unassigned). ${ }^{(2)}$ Among them, only HEV-3 and HEV-4 have been reported in humans and animals worldwide, and they are known to cause cross-species infection, posing a threat of zoonotic transmission. ${ }^{(3)}$ In Brazil, HEV-3 has been detected in humans and in swine in the South, Southeast, and Central-West regions. ${ }^{(4,5,6,7,8)}$ To date, HEV isolates have not been detected in Northeast Brazil. Swine production in this area is characterised by a low technical level and by poor investments in mechanisation, technology, and biosecurity practices. In this study, we screened for and performed phylogenetic analyses based on different genome segments of HEV strains circulating among domestic swine in Northeast Brazil.

A total of 119 faecal samples were individually collected in 2017 from two to six-month-old animals from eight farms using both intensive and extensive production systems located within a high HEV seroprevalence area. ${ }^{(9)}$ Viral RNA was extracted from a $200 \mu \mathrm{L}$ faecal

doi: 10.1590/0074-02760180585

Financial support: CNPq, FACEPE.

EFO-F and RD-C are supported by FACEPE and MCT/CNPq DCR grants.

+ Corresponding author: edmilsonz@gmail.com

(D) 0000-0002-3771-1565

Received 13 December 2018

Accepted 10 May 2019 suspension using the ReliaPrep ${ }^{\mathrm{TM}}$ Viral Total Nucleic Acid Purification Kit (Promega, Brazil) and screened by a previously described real-time polymerase chain reaction (RT-PCR) method ${ }^{(10)}$ using the NextGeneration ECO One-Step HotStart RT-qPCR Kit (DNA Express Biotecnologia, Brazil). Conventional HEV RT-PCR, using a set of previously designed primers to amplify the ORF2 region, ${ }^{(11,12)}$ was used to confirm the positive samples. Primers for sequencing the complete capsid region were designed based on the pertinent literature ${ }^{(13,14)}$ and on an alignment using 156 partial genomic Brazilian and HEV-3 subtype reference sequences (Tables I-II). RTPCR was performed using the SuperScript ${ }^{\mathrm{TM}}$ III OneStep RT-PCR System with Platinum ${ }^{\mathrm{TM}}$ Taq DNA Polymerase (Invitrogen, USA) according to manufacturer's instructions. Nested PCR was performed using PCR Master Mix 2X (Promega, Brazil), and the obtained PCR products were purified with the QIAquick Gel Extraction $\mathrm{Kit}^{\mathrm{TM}}$ (Qiagen) before being sent for sequencing.

Initially, sequences were aligned using MUSCLE v3.6 software. ${ }^{(15)}$ Markov models of nucleotide substitution were chosen using jMoldelTest v. 2 software. ${ }^{(16)}$ Sequences were retrieved from GenBank, and the accession numbers are displayed on the phylogenetic trees. Evolutionary studies were conducted using the maximum likelihood (ML) and maximum parsimony (MP) (17) inference methods implemented in FastTree v.2.1.7 $7^{(18)}$ and Mesquite v.3.5.1 $1^{(19)}$ software. ML analysis was conducted with the standard default GTR + CAT with 20 gamma distribution parameters and a mix of the nearest-neighbour interchanges (NNI) and sub-tree-prune- 
TABLE I

Oligonucleotides used to amplify the hepatitis E virus (HEV) capsid region

\begin{tabular}{|c|c|c|c|}
\hline Regions & Oligonucleotides & Obtained from & Genome position* \\
\hline Cap1 Ext F & GCGCAGGTYTGTGTTGATGT & This study & $4969-4988$ \\
\hline Cap1 Ext R & TACTGGGCATRGTTRGAYGCCTC & This study & $5702-5724$ \\
\hline Cap1 Int F & GGGYTGGTRCATAACCTYATTGG & This study & $5017-5039$ \\
\hline Cap1 Int R & GCCATAATRTGTGTRTTGGTGCC & This study & $5675-5697$ \\
\hline Cap2 Ext F & TCACCGGCCCCYGAYAC & This study & $5510-5526$ \\
\hline Cap2 Ext R & ARSCGRTGGCGGGCTGT & This study & $6146-6162$ \\
\hline Cap2 Int F & TGCGACGACAGTATAAYYT & This study & $5565-5583$ \\
\hline Cap2 Int $\mathrm{R}$ & GTRTACCGRGATACACG & This study & $6125-6141$ \\
\hline Cap3 Ext F & TGGTGATGCTYTGYATTCATGG & This study & $5994-6015$ \\
\hline Cap3 Ext R & ACCARTCMAGAGARCGGG & This study & $6702-6719$ \\
\hline CAP3 Int F & CTTGAYTTYGCGYTAGARCTTGA & This study & $6068-6089$ \\
\hline Cap3 Int R & CCTGRGCCCCTGTTGCYA & This study & $6678-6695$ \\
\hline Cap4 Ext F & GAGTAYGAYCAGACTACGTATGG & This study & $6605-6627$ \\
\hline Cap4 Int F & TCCACCAACCCGATGTATGT & This study & $6632-6651$ \\
\hline 15T-aTag & CCAACGACCGGGAGGCCATTTTTTTTTTTTTTTV & \multirow{2}{*}{ (14) } & Poly-A tail \\
\hline TAG & CCAACGACCGGGAGGCCA & & - \\
\hline
\end{tabular}

*: based on GenBank accession code AF082843.

regraft (SPR). The MP method, in which the best phylogenetic tree is determined based on favouring the fewest evolutionary changes, was performed using the parsimony ancestral states algorithm with the SPR rearranger to trace the history of character evolution on 1.000 trees. $^{(17)}$ The reliability of the tree nodes was obtained from Shimodaira-Hasegawa (SH-like) test support values with 1000 replications. ${ }^{(20)}$ Phylogenetic signals were assessed via likelihood mapping analysis using Tree-Puzzle v.5.2 software. ${ }^{(21,22)}$ The pairwise homoplasy index (PHI) for the Recombination search was implemented in SplitsTree v.4.14.6 following the default settings. ${ }^{(23)}$ Lastly, pairwise and patristic distances were inferred using MEGA v. $7^{(24)}$ and PATRISTIC ${ }^{(25)}$ software, respectively.

From a total of 119 samples, two samples (1.68\%) originating from three- (S67) and five-month-old (S26) clinically healthy piglets yielded 304-nucleotide (nt)-long DNA fragments corresponding to the $\mathrm{HEV}$ capsid region (Accession numbers: MH664123 and MH664124). The positive animals were from two distinct farms [Supplementary data (Fig. 2)]. Evolutionary analysis showed that both the S26 and S67 HEV sequences clustered within genotype HEV-3 together with the subtype $3 \mathrm{f}$ derived from a patient with acute hepatitis E in Japan who is known to have travelled to Bangladesh (see AB369387) (Fig. 1A). Additionally, we performed further analyses on a 1503-nt-long capsid sequence fragment isolated from sample S26. Analysis of this larger fragment along with $96 \mathrm{HEV}-3$ representative reference sequences retrieved using Boolean terms [Hepatitis E virus(Organism)] AND ORF2(Gene name) from GenBank, 43 of which had been previously assigned to clade $3 \mathrm{e}-3 \mathrm{f}-3 \mathrm{~g}$, thus confirming S26 to be phylogenetically related to clade $3 f$ (see Fig. 1C clade two highlighted by the dotted lines, SH-like support $\geq$ $80 \%$ ). These data were extrapolated from the consistent monophyletic origin and asymmetric phylogenetic tree backbone support [Fig. 1B, Supplementary data (Fig. 1)]. Intriguingly, although the sequence exhibited a high divergence, as demonstrated by the length of the tree branch (red line), we observed a proximity to the data obtained from the pairwise and patristic distances of HEV isolated from humans (KT591533.1 and KT591532.1) (Fig. 1B, Table II). Further gene flow analysis of S26 showed an ancestry degree and viral gene stream among HEV-3 virus populations typically found in human and animal hosts, suggesting both a switch of host direction and that this HEV-3 subtype $3 \mathrm{f}$ strain is probably of zoonotic origin (Fig. 1C). Similar patterns were also observed among strains of the HEV-e-f-g monophyletic clade, revealing a continuous flow of viral gene interchange and spread among sequences from human and animal hosts.

Although a human virus was recently suggested to be the most likely ancestor of Orthohepevirus A, ${ }^{(26)}$ the human or animal origin of HEV remains under debate. In addition, with regard to its progressive host range expansion, it appears that host switching has played an important role during the evolutionary history of $\mathrm{HEV}$ and that genotype differences might have arisen via cyclical adaptation to different hosts. ${ }^{(27)}$ Although there is a clear host range distinction among $\mathrm{HEV}$ genotypes, HEV-3 and HEV-4 viruses appear to be circulating between animal and human hosts. 
TABLE II

Sequences comparison among hepatitis E virus 3 (HEV-3) e-f-g subtypes extracted from the well-supported monophyletic clade highlighted in Fig. 1

\begin{tabular}{|c|c|c|c|c|c|c|c|}
\hline & Accession numbers & Countries & Hosts & Collection dates & Subtypes & Pairwise distances & Patristic distances \\
\hline 1 & AF455784 & Kyrgyzstan & Swine & 1987-89(2004) & $3 g$ & 0.226 & 0.3739 \\
\hline 2 & JQ013795 & France & Human & 2006 & $3 e^{\S}$ & 0.215 & 0.3339 \\
\hline 3 & AB291958 & Japan & Human & 2004 & $3 e$ & 0.203 & 0.3607 \\
\hline 4 & AB780453 & Japan & Wild boar & 2011 & $3 e$ & 0.218 & 0.3757 \\
\hline 5 & AB248521 & Japan & Swine & $2006^{*}$ & $3 e$ & 0.188 & 0.3497 \\
\hline 6 & AB248522 & Japan & Swine & 2006 & $3 e$ & 0.187 & 0.3537 \\
\hline 7 & JQ026407 & Japan & Monkey ${ }^{\S}$ & 2009 & $3 e$ & 0.211 & 0.3667 \\
\hline 8 & КР698919 & Italy & Swine & 2012 & $3 e$ & 0.206 & 0.3575 \\
\hline 9 & KF922359 & France & Human & $2009-10$ & $3 e$ & 0.212 & 0.3691 \\
\hline 10 & JQ953665 & France & Swine & 2006 & $3 e$ & 0.214 & 0.3518 \\
\hline 11 & FJ998015 & Germany & Wild boar & 2007 & $3 e$ & 0.200 & 0.3505 \\
\hline 12 & HM055578 & Hungary & Swine & 2005 & $3 e$ & 0.202 & 0.3278 \\
\hline 13 & AB290313 & Mongolia & Swine & 2006 & 3 & 0.190 & 0.2762 \\
\hline 14 & EU723512 & Spain & Swine & 2009 & 3 & 0.189 & 0.2468 \\
\hline 15 & KJ873911 & Germany & Human & 2013 & 3 & 0.177 & 0.2695 \\
\hline 16 & KT581447 & Sweden & Swine & $2015^{*}$ & 3 & 0.184 & 0.2861 \\
\hline 17 & KT581444 & Sweden & Swine & $2015^{*}$ & 3 & 0.161 & 0.2740 \\
\hline 18 & KT581446 & Sweden & Wild Boar & $2015^{*}$ & 3 & 0.167 & 0.2387 \\
\hline 19 & EU360977 & Sweden & Swine & $2007^{*}$ & 3 & 0.175 & 0.2633 \\
\hline 20 & KT581443 & Sweden & Swine & 2015 & 3 & 0.205 & 0.2810 \\
\hline 21 & KT581445 & Sweden & Wild boar & 2015 & 3 & 0.174 & 0.2832 \\
\hline 22 & EU723516 & Spain & Swine & $2008^{*}$ & 3 & 0.134 & 0.1790 \\
\hline 23 & EU723514 & Spain & Swine & $2008^{*}$ & 3 & 0.141 & 0.1788 \\
\hline 24 & EU723513 & Spain & Swine & $2008^{*}$ & 3 & 0.136 & 0.1767 \\
\hline 25 & LC164712 & Japan & Human & 2007 & 3 & 0.142 & 0.1953 \\
\hline 26 & LC055973 & Japan & Human & 2008 & 3 & 0.149 & 0.1832 \\
\hline 27 & AB850879 & Japan & Human & 2012 & 3 & 0.140 & 0.1874 \\
\hline 28 & EU495148 & France & Human & $2008^{*}$ & $3 \mathrm{f}$ & 0.139 & 0.1722 \\
\hline 29 & JN906975 & France & Swine & 2010 & $3 f$ & 0.111 & 0.1700 \\
\hline 30 & JN906974 & France & Human & 2010 & $3 f$ & 0.111 & 0.1700 \\
\hline 31 & JN906976 & France & Swine & 2010 & $3 f$ & 0.213 & 0.1700 \\
\hline 32 & KC166971 & France & Human & 2008 & $3 f$ & 0.114 & 0.1713 \\
\hline 33 & LC055972 & Japan & Human & 2012 & $3 f$ & 0.114 & 0.1827 \\
\hline 34 & KT581448 & Spain & Swine & $2015^{*}$ & $3 f$ & 0.118 & 0.1742 \\
\hline 35 & JQ953666 & France & Swine & 2008 & $3 f$ & 0.135 & 0.1499 \\
\hline 36 & FJ956757 & Germany & Human & 2005 & $3 f$ & 0.147 & 0.1899 \\
\hline 37 & KT591533 & France & Human & 2013 & $3 f$ & 0.124 & 0.1624 \\
\hline 38 & KT591532 & France & Human & 2013 & $3 f$ & 0.124 & 0.1624 \\
\hline 39 & KF891380 & Italy & Swine & $2013^{*}$ & $3 f$ & 0.131 & 0.1657 \\
\hline 40 & AB291961 & Japan & Human & 2004 & $3 f$ & 0.137 & 0.1810 \\
\hline 41 & EU375463 & Thailand & Swine & $2008^{*}$ & $3 \mathrm{f}$ & 0.141 & 0.1752 \\
\hline 42 & AB369687 & Japan $^{* *}$ & Human & 1998 & $3 f$ & 0.123 & 0.1657 \\
\hline 43 & FJ653660 & Thailand & Human & 2008 & $3 f$ & 0.148 & 0.1753 \\
\hline 44 & S26 & Brazil & Swine & 2017 & $3 f$ & 0.000 & 0.0000 \\
\hline
\end{tabular}

HEV-3 subtypes references ${ }^{(2)}$ are in bold. *: collection date was not available and GenBank deposition year is displayed; **: patient traveled to Thailand $\S$ classified as $3 \mathrm{f}$ on the GenBank $\S \S$ probably of human origin. 


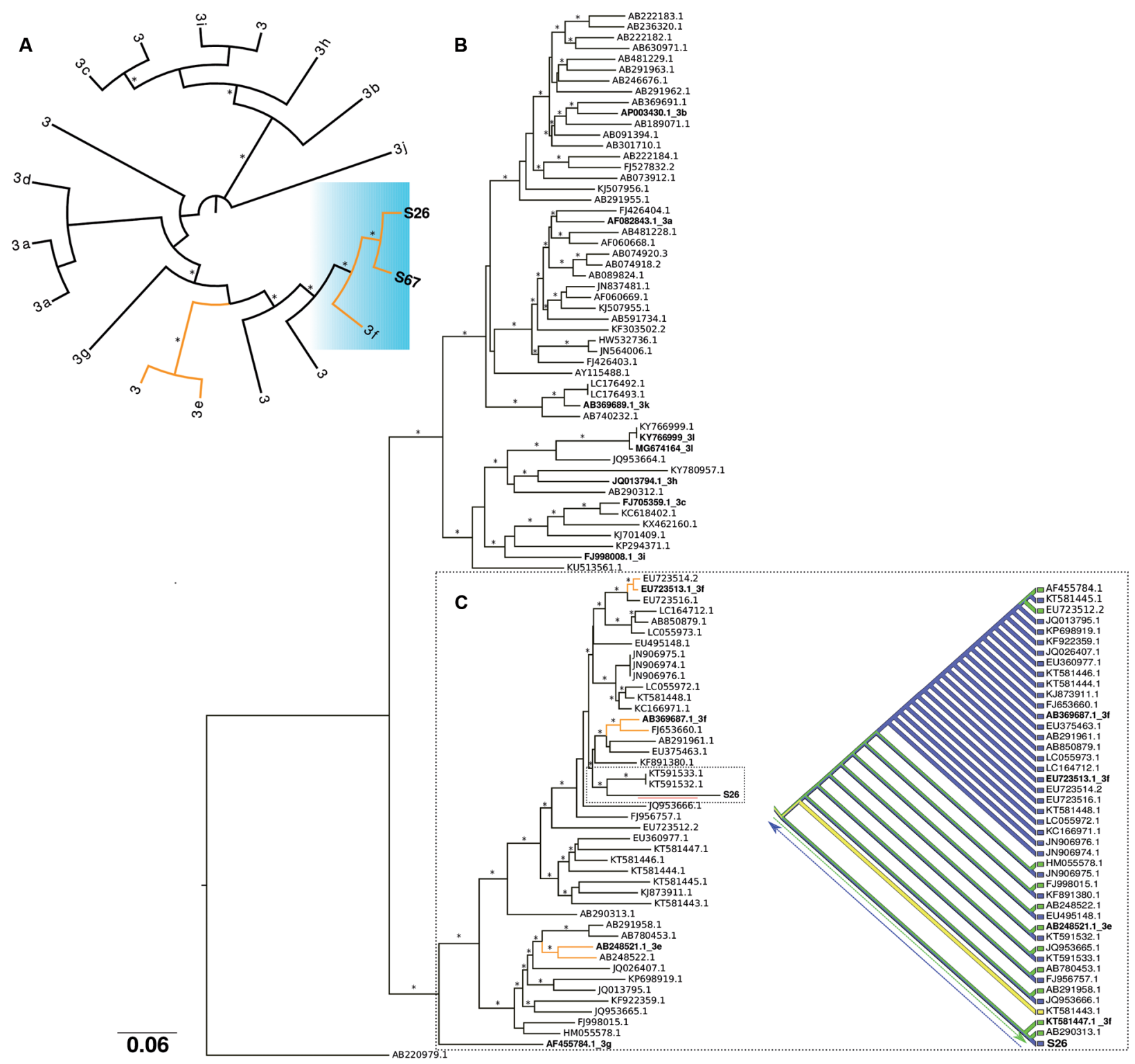

Phylogenetic analyses based on a $304 \mathrm{nt}$ fragment (A) and $1503 \mathrm{nt}$ (B and C) fragment of the hepatitis E virus 3 (HEV-3) capsid gene. Maximum likelihood (ML) phylogenetic tree showing the HEV samples in this study (blue gradient background: S26 and S69) clustering with subtype $3 \mathrm{f}$ isolated from humans (A). In (B), sequence S26 is in a rectangular dashed box. Subtype reference sequences ${ }^{(2)}$ are in bold. The red line under a tree branch highlights the sequence divergence according to the tree bar scale. The branches in orange colour highlight the HEV $3 \mathrm{f}$ and $3 \mathrm{e}$ clades. The asterisks along tree branches represent SH-like support values of $\geq 0.75$ (B). Parsimony ancestral states analysis inferring the character history of the sequences extracted from the second large clade from (B). Each colour represents HEV-3 isolated from different hosts, countries, and different sampling years (see Table II). The dotted blue and green arrows show our hypothesis about the continuous flow showing viral gene interchange and spread among HEV isolated from different hosts (C).

Our results are the first confirmation of HEV circulating among domestic swine farms in Northeast Brazil. The HEV RNA prevalence rate found here is markedly low considering the seroprevalence rates of up to $95 \%$ found in the same region. ${ }^{(9)}$ Nevertheless, our findings are similar to those obtained in other countries such as Germany, China, Japan, and India. ${ }^{(13,28,29,30)}$ Differences between serological and molecular prevalence rates are expected and likely to be related to animal age because HEV RNA is more easily detected in piglets that are two to six months old. ${ }^{(5)}$
Following the ICTV recommendation, ${ }^{(2)}$ S26 was assigned to subtype $3 \mathrm{f}$, which has already been detected in Brazil.(7) Although we were able to assign it successfully, a clear subtype separation based on pairwise or patristic distances was not possible (Table II). This divergence is likely related to differences in HEV-3 evolutionary rates among different subtypes, which seem to be higher among human-associated lineages. ${ }^{(27)}$ In addition, the marked heterogeneity observed within the subtype $3 \mathrm{f}$ and $3 \mathrm{e}-3 \mathrm{f}-3 \mathrm{~g}$ clade might be an evolutionary hallmark, for example resulting from the accumulation of mutations 
positively selected through successful infection in different hosts. ${ }^{(27)}$ Thus, these results illustrate that subtyping inside this clade can be challenging and might indicate the possibility of the future emergence of new subtypes if additional new heterogenic sequences appear.

The close relationship between HEV strains isolated from different host species points towards interspecies transmission. In addition, the gene flow study corroborated a possible evolutionary host switch origin and zoonotic potential. Thus, the molecular evidence indicates that the HEV strains identified here are potentially zoonotic and pose a threat regarding the infection of animal handlers, veterinarians, and consumers of non-cooked swine meat and pork products. Future studies should address HEV molecular epidemiology and explore the genetic variability and potential transmission among human and animal populations in Brazil.

\section{ACKNOWLEDGEMENTS}

To Louisa Ludwig-Begall for the careful English proofreading of this manuscript.

\section{AUTHORS' CONTRIBUTION}

EFOF - Study conception and design, acquisition of data, analysis and interpretation of data, drafting of manuscript, critical revision; DRLS and RD-C - analysis and interpretation of data, drafting of manuscript, critical revision; AS, GBL, AFBBF and LJP - study conception and design, acquisition of data, critical revision; LHVGG - study conception and design, analysis and interpretation of data, drafting of manuscript, critical revision.

\section{REFERENCES}

1. Purdy MA, Harrison TJ, Jameel S, Meng XJ, Okamoto H, Van der Poel WHM, et al. ICTV virus taxonomy profile: hepeviridae. J Gen Virol. 2017; 98(11): 2645-6.

2. Smith DB, Simmonds P, Izopet J, Oliveira-Filho EF, Ulrich RG, Johne R, et al. Proposed reference sequences for hepatitis E virus subtypes. J Gen Virol. 2016; 97(3): 537-42.

3. Nan Y, Wu C, Zhao Q, Zhou EM. Zoonotic hepatitis E virus: an ignored risk for public health. Front Microbiol. 2017; 8: 2396.

4. dos Santos DL, de Oliveira-Filho EF, Pinto MA. Hepatitis E worldwide and in Brazil: review. J Vet Med An Sci. 2013; 20(3): 416-33.

5. dos Santos DR, Vitral CL, de Paula VS, Marchevsky RS, Lopes JF, Gaspar AM, et al. Serological and molecular evidence of hepatitis E virus in swine in Brazil. Vet J. 2009; 182(3): 474-80.

6. dos Santos DR, de Paula VS, de Oliveira JM, Marchevsky RS, Pinto MA. Hepatitis E virus in swine and effluent samples from slaughterhouses in Brazil. Vet Microbiol. 2011; 149(1-2): 236-41.

7. Lopes Dos Santos DR, Lewis-Ximenez LL, da Silva MF, de Sousa PS, Gaspar AM, Pinto MA. First report of a human autochthonous hepatitis E virus infection in Brazil. J Clin Virol. 2010; 47(3): 276-9.

8. da Costa Lana MV, Gardinali NR, da Cruz RA, Lopes LL, Silva GS, Caramori Jr JG, et al. Evaluation of hepatitis E virus infection between different production systems of pigs in Brazil. Trop Anim Health Prod. 2014; 46(2): 399-404.

9. de Oliveira-Filho EF, Lopes KG, Cunha DS, Silva VS, Barbosa CN, Brandespim DF, et al. Risk analysis and occurrence of hepatitis E virus (HEV) in domestic swine in Northeast Brazil. Food Environ Virol. 2017; 9(3): 256-9.

10. Jothikumar N, Cromeans TL, Robertson BH, Meng XJ, Hill VR. A broadly reactive one-step real-time RT-PCR assay for rapid and sensitive detection of hepatitis E virus. J Virol Methods. 2006; 131(1): 65-71.
11. Meng XJ, Purcell RH, Halbur PG, Lehman JR, Webb DM, Tsareva $\mathrm{TS}$, et al. A novel virus in swine is closely related to the human hepatitis E virus. Proc Natl Acad Sci USA. 1997; 94(18): 9860-5.

12. Huang FF, Haqshenas G, Guenette DK, Halbur PG, Schommer SK, Pierson FW, et al. Detection by reverse transcription-PCR and genetic characterization of field isolates of swine hepatitis $E$ virus from pigs in different geographic regions of the United States. J Clin Microbiol. 2002; 40(4): 1326-32.

13. Oliveira-Filho EF, Bank-Wolf BR, Thiel HJ, König M. Phylogenetic analysis of hepatitis $\mathrm{E}$ virus in domestic swine and wild boar in Germany. Vet Microbiol. 2014; 174(1-2): 233-8.

14. Müller B, Klemm U, Mas Marques A, Schreier E. Genetic diversity and recombination of murine noroviruses in immunocompromised mice. Arch Virol. 2007; 152(9): 1709-19.

15. Edgar RC. MUSCLE: a multiple sequence alignment method with reduced time and space complexity. BMC Bioinformatics. 2004; 5: 113-29.

16. Darriba D, Taboada GL, Doallo R, Posada D. jModelTest 2: more models, new heuristics and parallel computing. Nat Methods. 2012; 9(8): 772-5.

17. Moratorio G, Vignuzzi M. Monitoring and redirecting virus evolution. PLoS Pathog. 2018; 14(6): e1006979.

18. Price MN, Dehal PS, Arkin AP. FastTree 2 - approximately maximum-likelihood trees for large alignments. PLoS One. 2010; 5(3): e9490.

19. Maddison WP, Maddison DR. Mesquite: a modular system for evolutionary analysis. Version 3.512018 [cited 2018 30.09.2018]. Available from: http://www.mesquiteproject.org.

20. Anisimova M, Gascuel O. Approximate likelihood-ratio test for branches: a fast, accurate, and powerful alternative. Syst Biol. 2006; 55(4): 539-52.

21. Schmidt HA, Strimmer K, Vingron M, von Haeseler A. TREEPUZZLE: maximum likelihood phylogenetic analysis using quartets and parallel computing. Bioinformatics. 2002; 18(3): 502-04.

22. Strimmer K, von Haeseler A. Likelihood-mapping: a simple method to visualize phylogenetic content of a sequence alignment. Proc Natl Acad Sci USA. 1997; 94(13): 6815-9.

23. Huson DH, Bryant D. Application of phylogenetic networks in evolutionary studies. Mol Biol Evol. 2006; 23(2): 254-67.

24. Kumar S, Stecher G, Tamura K. MEGA7: Molecular Evolutionary Genetics Analysis Version 7.0 for bigger datasets. Mol Biol Evol. 2016; 33(7): 1870-4.

25. Fourment M, Gibbs MJ. PATRISTIC: a program for calculating patristic distances and graphically comparing the components of genetic change. BMC Evol Biol. 2006; 6: 1.

26. Forni D, Cagliani R, Clerici M, Sironi M. Origin and dispersal of hepatitis E virus. Emerg Microbes Infect. 2018; 7(1): 11.

27. Brayne AB, Dearlove BL, Lester JS, Pond SLK, Frost SD. Genotype-specific evolution of hepatitis E virus. J Virol. 2017; 91(9): pii: e02241-16.

28. Geng Y, Wang C, Zhao C, Yu X, Harrison TJ, Tian K, et al. Serological prevalence of hepatitis $\mathrm{E}$ virus in domestic animals and diversity of genotype 4 hepatitis $\mathrm{E}$ virus in China. Vector Borne Zoonotic Dis. 2010; 10(8): 765-70.

29. Sakano C, Morita Y, Shiono M, Yokota Y, Mokudai T, Sato-Motoi $\mathrm{Y}$, et al. Prevalence of hepatitis E virus (HEV) infection in wild boars (Sus scrofa leucomystax) and pigs in Gunma Prefecture, Japan. J Vet Med Sci. 2009; 71(1): 21-5.

30. Vivek R, Kang G. Hepatitis E virus infections in swine and swine handlers in Vellore, Southern India. Am J Trop Med Hyg. 2011; 84(4): 647-9. 